\title{
Cerebral edema in a patient following cytoreductive surgery and hyperthermic intraoperative intraperitoneal chemoperfusion Rajalakshmi L Nair ${ }^{1}$, Jonathan Tobias ${ }^{2}$, Grant Stemmerman ${ }^{2}$ and Andrew M Lowy*1
}

\author{
Address: ${ }^{1}$ Departments of Surgery, Division of Surgical Oncology, University of Cincinnati, Cincinnati, OH 45267, USA and ${ }^{2}$ Department of \\ Pathology and Laboratory Medicine, University of Cincinnati, Cincinnati, OH 45267, USA \\ Email: Rajalakshmi L Nair - nair_rl@yahoo.com; Jonathan Tobias - jonathan.tobias@uc.edu; Grant Stemmerman - grant.stemmerman@uc.edu; \\ Andrew M Lowy* - andrew.lowy@uc.edu \\ * Corresponding author
}

Published: 30 November 2006

World Journal of Surgical Oncology 2006, 4:85 doi:10.1 186/1477-78/9-4-85

This article is available from: http://www.wjso.com/content/4/l/85

(C) 2006 Nair et al; licensee BioMed Central Ltd.

This is an Open Access article distributed under the terms of the Creative Commons Attribution License (http://creativecommons.org/licenses/by/2.0), which permits unrestricted use, distribution, and reproduction in any medium, provided the original work is properly cited.
Received: 30 June 2006

Accepted: 30 November 2006

\begin{abstract}
Background: Cytoreductive surgery and intraoperative, intraperitoneal hyperthermic chemoperfusion (HIPEC) is increasingly used to treat peritoneal surface metastases. We describe a fatal case of cerebral edema in a patient with appendiceal carcinoma and an underlying seizure disorder who underwent cytoreductive surgery and HIPEC.
\end{abstract}

Case presentation: A case of fatal postoperative cerebral edema is presented in a patient with an underlying seizure disorder and recurrent mucinous adenocarcinoma of the appendix. The patient was treated with cytoreductive surgery and intraoperative intraperitoneal hyperthermic chemoperfusion. The details and implications of this complication are discussed.

Conclusion: The recognition of this potential complication is important for physicians performing cytoreductive surgery and HIPEC. Special caution should be taken when patients with seizure disorders are being considered for this treatment.

\section{Background}

The treatment of peritoneal metastases remains one of the most challenging problems in clinical oncology. Over the last decade, interest in the use of aggressive cytoreductive surgery combined with hyperthermic intraperitoneal intraoperative chemotherapy (HIPEC) has increased [1]. This interest has been fueled by data from single institutions and collected series demonstrating long-term survival for selected patients with peritoneal surface metastases treated with this combined modality approach [2]. Along with the availability of new cytotoxic and biologic therapies for gastrointestinal cancers, recent data demonstrating improved survival for ovarian cancer patients treated with postoperative intraperitoneal therapy has only served to increase the interest in such treatment for patients with GI malignancies [3-6].

To date, there has been only a single large randomized study, which demonstrated a benefit for patients with colon carcinoma treated with cytoreductive surgery, HIPEC and systemic chemotherapy versus palliative surgery and chemotherapy alone [3]. Not surprisingly, the most successful outcomes for cytoreduction and HIPEC have been achieved in patients with low-grade malignancies of the appendix [7]. These tumors have a propensity to metastasize solely to peritoneal surfaces, and thus are 
an ideal tumor type to apply aggressive surgery and intraperitoneal therapies. Along with tumor grade, the completeness of cytoreduction appears to most strongly correlate with patient outcome [8]. Current practice for HIPEC involves either an open abdomen or closed abdomen into which the chemotherapeutic agent(s) are heated to approximately $41^{\circ} \mathrm{C}$. Mitomycin and cisplatin are the most commonly used chemotherapeutic agents. HIPEC using these agents results in major morbidity in approximately one third of patients and mortality rates range from $0-12 \%$ in recent series $[9,10]$. Common complications include intraabdominal abscess/fistula, pulmonary embolism and neutropenia. Here we present a case of death secondary to cerebral edema in a patient who underwent cytoreductive surgery + HIPEC for recurrent appendiceal carcinoma with Mitomycin C.

\section{Case presentation}

A 37 year old female presented to her primary care physician with nonspecific abdominal pain. She underwent CT imaging that revealed bilateral ovarian masses and ascites. Preoperative CA125 was the only tumor marker examined and it was within normal limits. A presumptive diagnosis of ovarian cancer was made and she underwent exploratory laparotomy which revealed macroscopic peritoneal metastases to ovaries, omentum and pelvic peritoneum. At that time total abdominal hysterectomy, bilateral salpingoopherectomy, pelvic lymph node sampling, partial omentectomy, appendectomy and evacuation of mucinous ascites was performed. Final pathology revealed low grade mucinous adenocarcinoma in all specimens, arising from an appendiceal primary. The patient received no additional therapy and was followed clinically. She developed recurrent abdominal pain approximately 2 years later. Computed tomography (CT) scan revealed progressive nodularity in the mesentery and peritoneal surfaces consistent with recurrent disease. This was further evaluated with a laparoscopy, which revealed mucinous tumor implants along the anterior abdominal wall, right retroperitoneum, diaphragm, and remaining omentum (Figure $1 \mathrm{~A}, \mathrm{~B})$. At this time, the patient was referred to the University of Cincinnati for consideration of cytoreductive surgery and intraperitoneal hyperthermic chemoperfusion. The patient's past medical history was significant only for a seizure disorder which was managed by anticonvulsant medication and an implanted vagus nerve stimulator. Preoperative CA19-9 was elevated at 59, while CEA and CA-125 were normal. The patient was felt to be an appropriate candidate and underwent exploration. At surgery, extensive disease was noted over the peritoneal surfaces. A complete cytoreduction was achieved requiring, right colectomy, splenectomy, cholecystectomy, omentectomy, and perionectomies of the diaphragms, anterior abdominal wall, and pelvis. Tumor nodules were excised from the small bowel and large bowel mesentery.
The cytoreductive portion of the operation was completed in approximately 210 minutes. Per our current practice protocol, a 90 minute perfusion was performed using an inflow temperature of $44^{\circ} \mathrm{C}$, with peritoneal surface temperatures averaging $40.5-41.5^{\circ} \mathrm{C}$. Intraperitoneal temperatures were measured via four temperature probes, one within the liver parenchyma, and three within separate quadrants of the peritoneum. Core temperature was recorded via the bladder and esophageal temperature probes. Per our standard protocol, the carrier solution was peritoneal dialysis fluid $(2.5 \%)$. The patient's maximum recorded core body temperature during the procedure was $38.7^{\circ} \mathrm{C}$ and the maximum recorded liver temperature was $38.8^{\circ} \mathrm{C}$. Mitomycin $\mathrm{C}$ was delivered using a total dose of $10 \mathrm{mg} / \mathrm{L}$ perfusate, delivered in divided doses of $7 \mathrm{mg} / \mathrm{L}$ at initiation and redosed after 45 minutes at $3 \mathrm{mg} / \mathrm{L}$. A total dose of $45 \mathrm{mg}$ was delivered in this patient. During the operation, the patient had no significant electrolyte abnormalities aside from the expected glucose elevation seen during the perfusion period. The maximum serum glucose was $355 \mathrm{mg} / \mathrm{dL}$ and the lowest serum sodium was 135. The operation was generally uneventful; no blood transfusions were required and the patient was transferred to the ICU in stable condition. Postoperatively, all serum electrolytes were normal and albumin was $3.4 \mathrm{mg} / \mathrm{dl}$ compared with $4.3 \mathrm{mg} / \mathrm{dl}$, preoperatively. The patient was easily arousable and followed commands appropriately. However, approximately 4 hrs later, the patient complained of headache and right eye pain. Her headache was treated with analgesics, however 3 hours later, she was found to be tachypenic, bradycardic, and hypotensive, and was unresponsive with fixed, dilated pupils. She was emergently intubated and resuscitated. After approximately 15 minutes, she regained consciousness, and responded appropriately to commands. Her pupils returned to $3 \mathrm{~mm}$ and because reactive. Neurology was consulted and she was loaded with Dilantin due to her history of seizure disorder. An urgent CT scan was ordered. On route to the CT scanner, she once again became bradycardic and hypotensive with fixed, dilated pupils. The CT scan revealed diffuse cerebral edema (Figure 2). ICP monitoring revealed pressures of 70-90 $\mathrm{mmHg}$. Mannitol was used in an attempt to lower intracranial pressure without success. EEG was consistent with diffuse brain dysfunction. A brain death protocol revealed that she had suffered brain death and care was subsequently withdrawn. At autopsy, diffuse cerebral edema with tonsillar herniation was noted (Figure 3). No pathologic findings were present to indicate the cause of the cerebral edema. The final pathology on all resected specimens reconfirmed the diagnosis of mucinous adenocarcinoma (Figure 4A,B). 

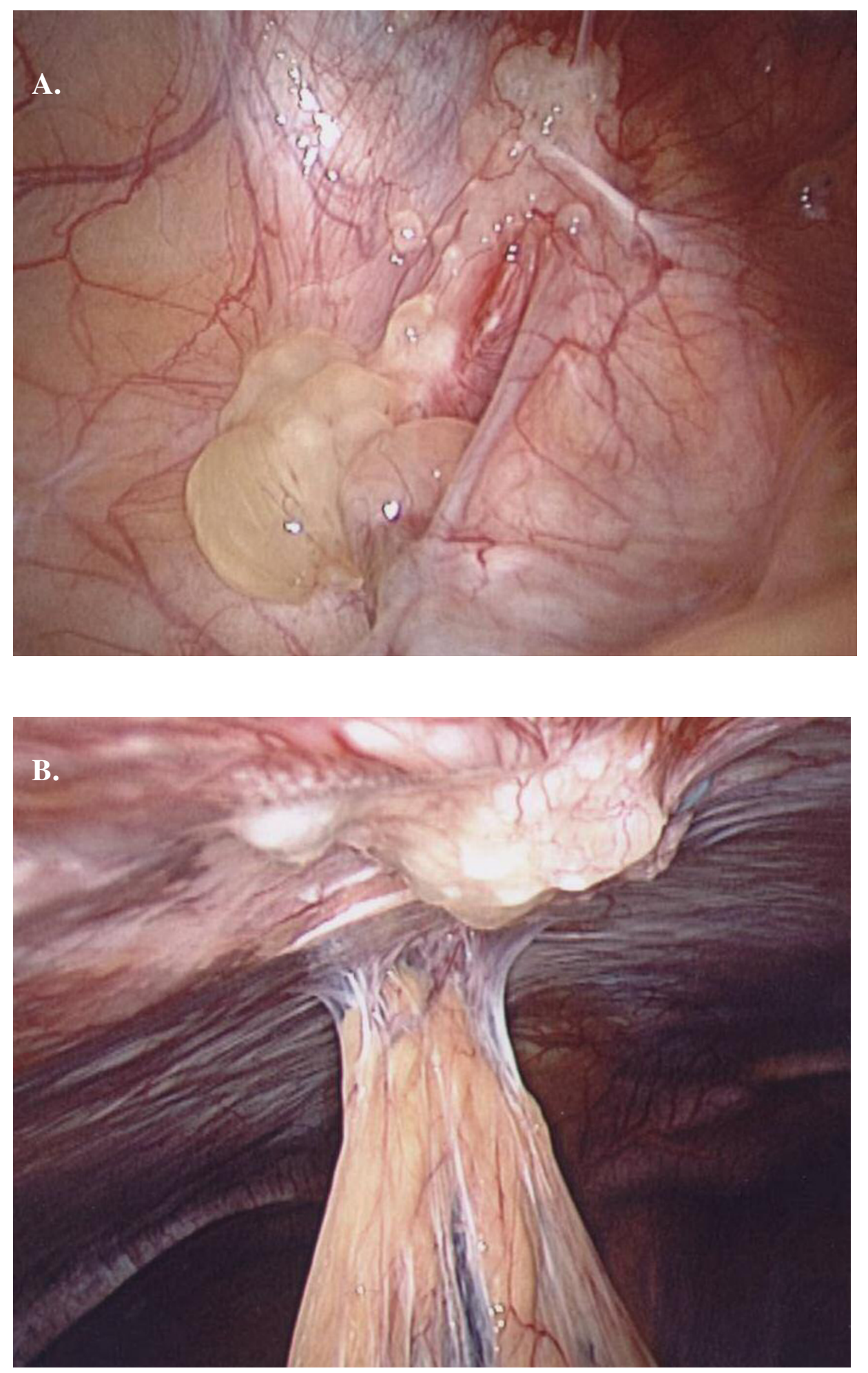

Figure I

A) Laparoscopic view of the cecum demonstrating foci of recurrent appendiceal mucinous tumor. B) Laparoscopic view of the anterior abdominal wall demonstrating recurrent mucinous tumor adherent to the parietal peritoneum. Note evidence of more solid tumor deposits suggesting the presence of adenocarcinoma as opposed to simple adenomucinosis. 


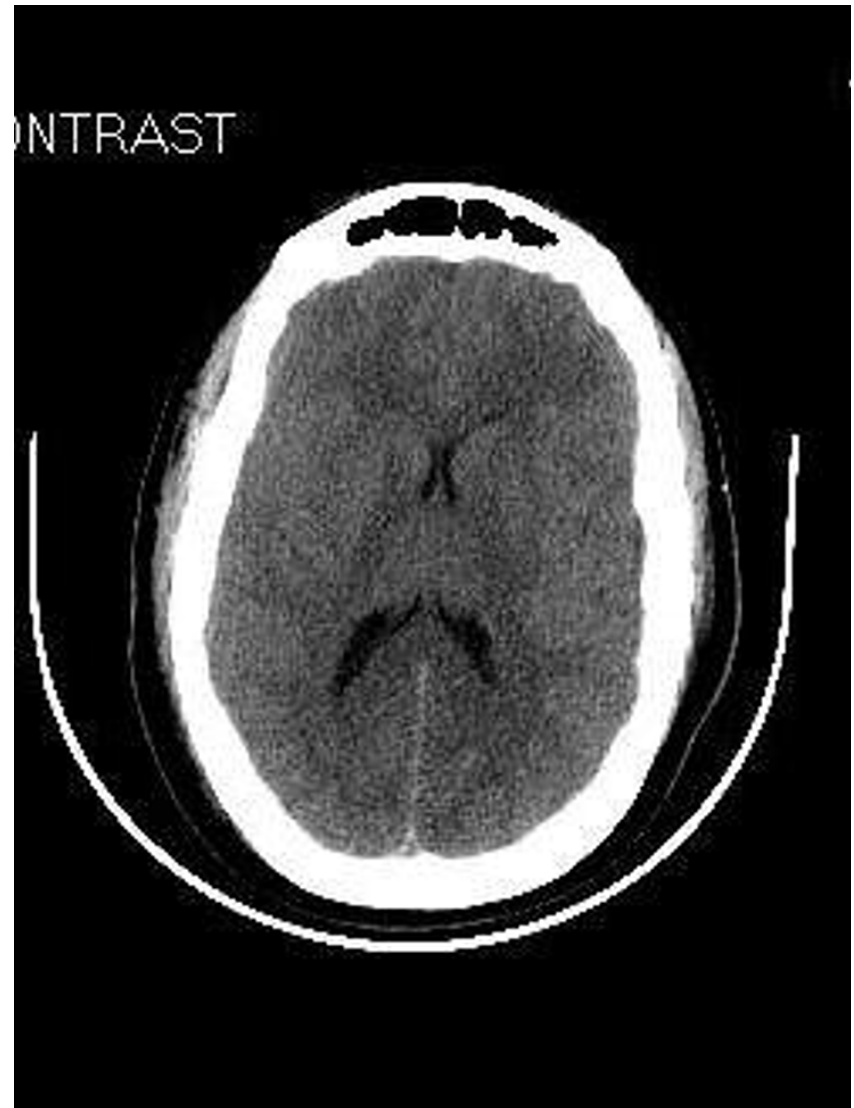

Figure 2

Diffuse cerebral edema resulting in compression of ventricles.

\section{Discussion}

Studies of HIPEC have revealed characterized several alterations in the physiology of patients during this treatment. Core body temperature is seen to increase significantly, but can usually be maintained in acceptable range by precooling and/or through the use of external cooling measures. Heart rate, CVP and PAP also increase, yet MAP, CO, oxygen delivery, and oxygen consumption do not change significantly $[11,12]$. Transient hyperglycemia occurs presumably secondary both to the stress response from surgery and hyperthermia, and to the presence of dextrose in the perfusate. In our patient, electrolytes remained within the normal range. The expected transient hyperglycemia responded appropriately to insulin. Core temperature peaked at $38.7^{\circ} \mathrm{C}$ and subsequently returned to normal. These are not likely to have contributed to the acute cerebral edema that she suffered.

Seizures are temporary abnormal electrophysiologic phenomena of the brain that result in abnormal synchronization of electrical neuronal activity. They can manifest as an alteration in mental state, tonic or clonic movements and various other symptoms.

When seizure activity is continuous it is referred to as status epilepticus. This persistent condition can lead to neuronal injury. Generalized convulsive status epilepticus (GCSE) is the most common form and is associated with a significant risk of mortality. All other forms of status epilepticus are grouped as nonconvulsive status epilepticus. Etiologies may include high fever and infection in children, electrolyte abnormalities, previous CNS insult, and hypoxia [13].

Acute brain swelling occurs is seen in a variety of clinical settings. Systemic complications of brain swelling include fever, lactic acidosis, hyperglycemia, respiratory difficulties, and cardiovascular changes $[14,15]$. In one animal study, persistent seizures resulted in generalized cerebral edema at 2 hours and necrotic edema at 24 hours [15]. Acute cerebral edema has been documented after generalized convulsive status epilepticus. Our patient suffered from complex partial seizures. We were unable to find evidence of cerebral edema occurring in a patient with complex partial epilepticus. In a case report, transient seizureinduced MR enhancement consistent with ictal or postictal hyperemia in patients with complex partial seizures was reported. However, there changes were noted to be transient [16]. Despite the absence of reports, it remains a possibility that in this patient, acute cerebral edema may have occurred secondary to unrecognized partial complex seizures in a patient whose seizure threshold may have been lowered secondary to the stress of surgery and hyperthermia. Patients with documented complex partial seizure may have confusion as the only manifestation of their seizure activity and thus it is possible to miss the only clinical signs. However, the postoperative EEG in our patient showed no evidence of status epilepticus, thus arguing against this explanation. Additionally, there have been no reports of any association between the presence of a vagus nerve stimulator and acute cerebral edema.

Hyperthermia is reported to predispose the brain to hypoxic/anoxic injury. In our patient, systemic hyperthermia never reached critical levels and had resolved postoperatively. There were no instances of hypoxia in this patient. Thus, unfortunately the etiology of the cerebral edema remains unclear. Given the increasing use of HIPEC, we believe the documentation of unusual complications such as this is important in the consideration of operative risk and for informed consent in future patients with peritoneal surface metastases.

As a result of this event, our group has now incorporated preoperative consultation with a neurologist to insure adequacy of preoperative anticonvulsant therapy and to 


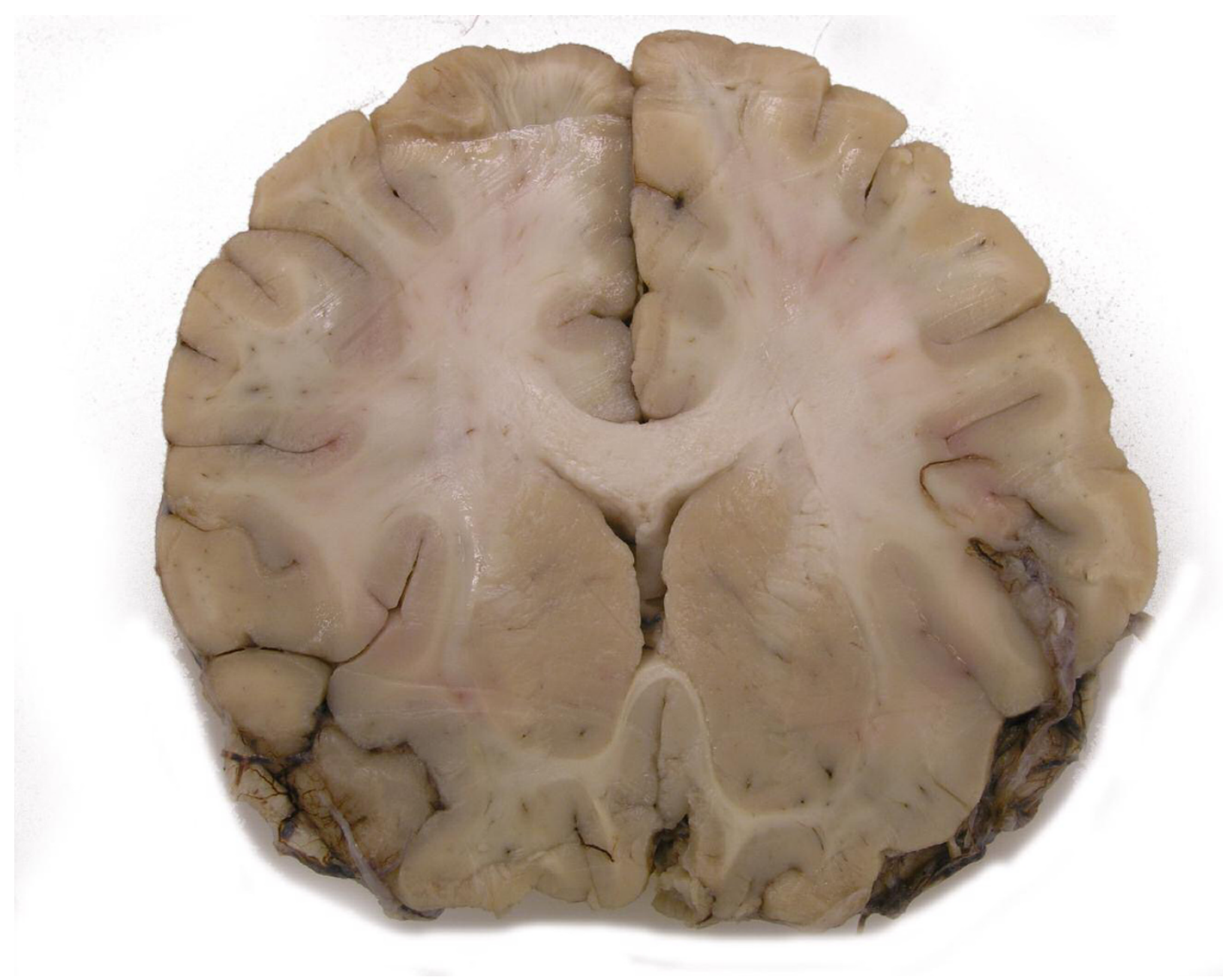

Figure 3

Coronal section demonstrating normal grey and white matter with marked loss of luminal dimension in the ventricular system due to diffuse cortical edema.

comment on the possible value of intraoperative EEG monitoring prior to considering HIPEC in any patient with a history of seizures.

\section{Conclusion}

This article represents the first description of fatal cerebral edema following treatment of appendiceal carcinoma with intraperitoneal hyperthermic chemoperfusion. This complication occurred in a patient with an underlying seizure disorder and raises the question as to whether this may have been a causative factor. The recognition of this potential complication is important for physicians performing cytoreductive surgery and HIPEC. Special caution should be taken when patients with seizure disorders are being considered for this treatment.

\section{Conflict of interest}

The author(s) declare that they have no competing interests.

\section{Authors' contributions}

RLN participated in manuscript writing and figure preparation. JT and GS performed the pathologic analyses, assisted with manuscript editing and figure preparation. AML conceived the report, participated in manuscript writing and edited the manuscript. All authors read and approved the final manuscript. 

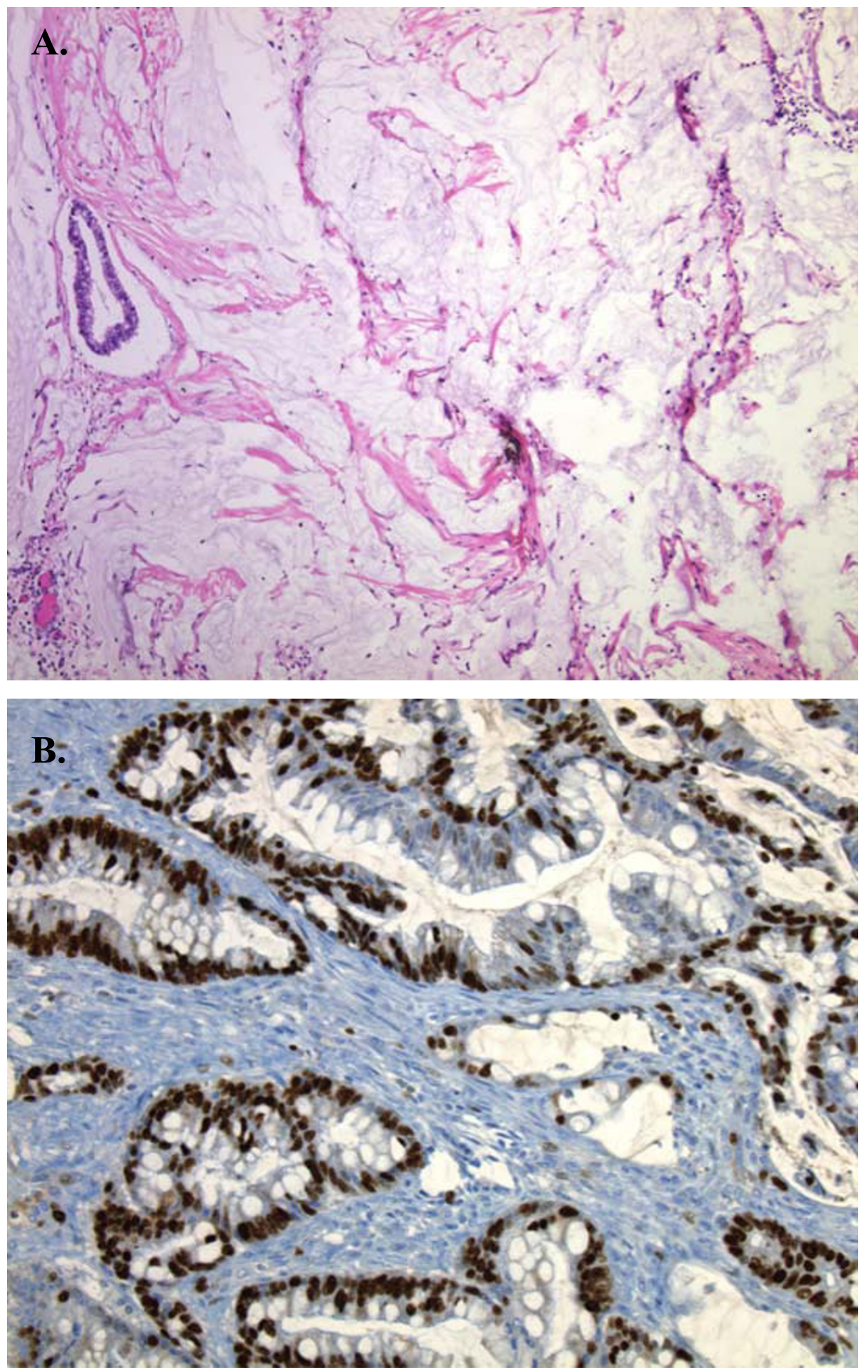

Figure 4

A) Foci of adenocarcinoma from the right colon demonstrating large amounts of mucin on the serosal surface. B) Multiple foci of mucinous adenocarcinoma with Mib-I staining, indicating a high proliferative rate. 


\section{Acknowledgements}

Patients consent was obtained for publication of this case report

\section{References}

I. Stewart JH IV, Shen P, Levine EA: Intraperitoneal chemotherapy for peritoneal surface malignancy: current status and future directions. Ann Surg Oncol 2005, I 2:765-777.

2. Glehen O, Kwiatkowski M, Sugarbaker PH, Elias D, Levine EA, Gilly FN, De Simone M, Barone R, Yonemura Y, Cavaliere F, Quenet F, Gutman M, Tentes AAK, Lorimier G, Bernard JL, Bereder JM, Porcheron J, Gomez-Portilla A, Shen P, Deraco M, Rat P: Cytoreductive surgery combined with perioperative intraperitoneal chemotherapy for the management of peritoneal carcinomatosis from colorectal cancer: a multi-institutional study. J Clin Oncol 2004, 22:3284-3292.

3. Armstrong DK, Bundy B, Wenzel L, Huang HQ, Baergen R, Lele S, Copeland LJ, Walker JL, Burger RA, Gynecologic Oncology Group: Intraperitoneal cisplatin and paclitaxel in ovarian cancer. $N$ Engl J Med 2006, 354:34-43.

4. Hamilton CA, Berek JS: Intraperitoneal chemotherapy for ovarian cancer. Curr Opin Oncol 2006, I8:507-5I5.

5. Verwaal VJ, van Ruth S, de Bree E, van Sloothen GW, van Tinteren $H$, Boot $\mathrm{H}$, Zoetmulder FA: Randomized trial of cytoreduction and hyperthermic intraperitoneal chemotherapy versus systemic chemotherapy and palliative surgery in patients with peritoneal carcinomatosis of colorectal cancer. J Clin Oncol 2003, 2 I:3737-3743.

6. Jacks SP, Hundley JP, Shen P, Russell GB, Levine EA: Cytoreductive surgery and intraperitoneal hyperthermic chemotherapy for peritoneal carcinomatosis from small bowel adenocarcinoma. J Surg Oncol 2005, 91 : I I2-7.

7. Brigand C, Arvieux C, Gilly FN, Glehen O: Treatment of peritoneal carcinomatosis in gastric cancers. Dig Dis 2004, 22:366-373.

8. Sugarbaker $\mathrm{PH}$, Chang D: Results of treatment of $\mathbf{3 8 5}$ patients with peritoneal surface spread of appendiceal malignancy. Ann Surg Oncol 1999, 6:727-731.

9. Ahmad SA, Sussman J, Kim J, Soldano DA, Pennington L, Lowy AM: Reduced morbidity and mortality following cytoreductive surgery and intraperitoneal hyperthermic perfusion. Ann Surg Oncol 2004, I I:387-392.

10. Smeenk RM, Verwaal VJ, Zoetmulder FA: Toxicity and mortality of cytoreduction and intraoperative hyperthermic intraperitoneal chemotherapy in pseudomyxoma peritonei - a report of 103 procedures. Eur J Surg Oncol 2006, 32:186-190.

II. Kanakoudis F, Petrou D, Michaloudis D, Chortaria G, Konstantinidou A: Anaesthesia for intraperitoneal perfusion of hyperthermic chemotherapy. Anaesthesia 1996, 51:1033-1036.

I2. Esquivel J, Angulo F, Bland RK, Stephens AD, Sugarbaker PH: Hemodynamic and cardiac function parameters during heated intraoperative intraperitoneal chemotherapy using the open "coliseum technique. Ann Surg Oncol 2000, 7:296-300.

13. Fountain NB: Status Epilepticus: Risk factors and complications. Epilepsia 2000, 4I:23-30.

14. Nelson SR, Olson JP: Role of early edema in the development of regional seizure-related brain damage. Neurochemical Research 1987, 1 2:561-564.

15. Seitelberger F, Lassmann H, Hornykiewicz O: Some mechanism of brain edema studies in a Kainic acid model. ACTA Neurobiol Exp 1990, 50:263-267.

16. Kim J, Chung J, Yoon PH, Kim DI, Chung TS, Kim EJ, Jeong EK: Transient MR signal changes in patients with generalized tonicoclonic seizure or status epilepticus: perictal diffusionweighted imaging. Am J Neurorad 200।, 22: I I49-I I60.

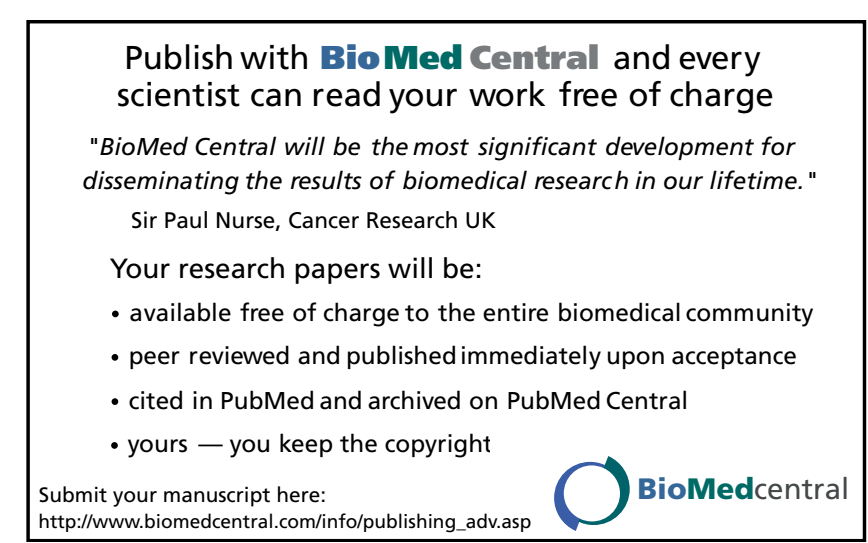

